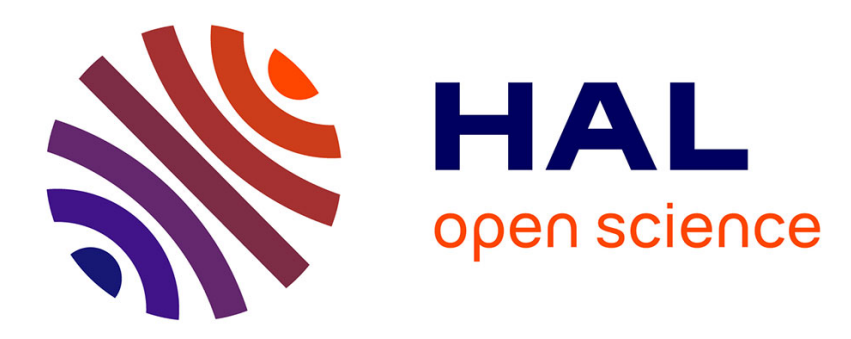

\title{
The risk of threshold responses, tipping points, and cascading failures in pollination systems
}

\author{
Tanya Latty, Vasilis Dakos
}

\section{To cite this version:}

Tanya Latty, Vasilis Dakos. The risk of threshold responses, tipping points, and cascading failures in pollination systems. Biodiversity and Conservation, 2019, 28 (13), pp.3389-3406. 10.1007/s10531019-01844-2 . hal-02321887

\section{HAL Id: hal-02321887 \\ https://hal.science/hal-02321887}

Submitted on 14 Dec 2020

HAL is a multi-disciplinary open access archive for the deposit and dissemination of scientific research documents, whether they are published or not. The documents may come from teaching and research institutions in France or abroad, or from public or private research centers.
L'archive ouverte pluridisciplinaire HAL, est destinée au dépôt et à la diffusion de documents scientifiques de niveau recherche, publiés ou non, émanant des établissements d'enseignement et de recherche français ou étrangers, des laboratoires publics ou privés. 


\title{
The risk of threshold responses, tipping points, and cascading
} failures in pollination systems

1. School of Life and Environmental Sciences, University of Sydney, Sydney, NSW 2006, Australia

2. Institut des Sciences de l'Evolution de Montpellier (ISEM), Université de Montpellier, CNRS, Montpellier, France

Corresponding author : Tanya.latty@sydney.edu.au, +61 293514361

\author{
Tanya Latty' and Vasilis Dakos
}

(3)

\section{Abstract}

0 Growing evidence of global declines in pollinator abundance and diversity has raised

1 concerns about the resilience of pollination systems. When subjected to stressors, each nested 42 component of the pollination system (communities, populations, and colonies) can respond in 43 either a smooth linear fashion, or in an abrupt nonlinear manner. Threshold and tipping point 
44 responses to stress are of particular concern because they result in sudden changes with little 45 warning; such changes may lead to persistent non-functional states that are difficult to 46 reverse. Here, we review evidence for threshold and tipping point responses at the colony, 47 population and community levels of the pollination system. We find that while there are 48 strong theoretical reasons to expect tipping point and threshold responses at all three levels of 49 the pollination system, evidence in the field is lacking for all levels except the colony level. 50 While this is encouraging, caution is still warranted as tipping point and threshold responses 51 - by their very nature- may not be apparent until they are underway. Moreover, we propose 52 that the interaction of nonlinear stress responses across different levels of the pollination 53 system can increase the risk of cascading failures. We therefore suggest a cautious approach 54 toward the management of pollination systems. Since environmental change will almost 55 certainly continue to accelerate, understanding the potential for thresholds, tipping points and 56 cascading failures is key to safeguarding global pollination systems.

58 Keywords: pollinator, plant-pollinator community, bee colony, nonlinear, interacting stress, 59 collapse 
61 Pollinating animals have long been recognized for their ecological and economic importance.

62 In temperate regions, $78 \%$ of flowering plants are animal pollinated; this number rises to $94 \%$

63 in tropical communities (Ollerton et al. 2011). The global economic value of animal

64 pollination is estimated at 153 billion Euros annually (Gallai et al. 2009) with approximately

$6575 \%$ of the world's major crops being moderately to totally dependent on animal pollination

66 (Klein et al. 2007). The past century has seen massive and accelerating levels of human-

67 induced environmental change, much of which directly or indirectly impacts pollinators and

68 the ecosystem services they provide. There is convincing evidence of ongoing global declines

69 in many pollinator taxa (Biesmeijer et al 2006, Carvalheiro et al 2013, reviewed in Potts et al 2010 and Sanchez-Bayo and Wyckhuys 2019).

Pollinators are assailed by an array of interacting stressors from habitat loss and alteration to disease, invasive species, poor nutrition, exposure to agrochemicals and climate change (reviewed in Vanbergen 2013, Goulson et al. 2015, Gonzalez-Varo et al 2013). In the short term it is unlikely that these stressors will decrease; indeed, many (such as habitat loss and climate change) are on course to continue into the foreseeable future. Safeguarding our pollination systems against this backdrop of accelerating global change requires an understanding of how pollination systems may respond to environmental stressors.

From a conservation perspective, it is not only the speed and magnitude of system response that is important, but also the shape of the response function. Responses to environmental stressors can be broadly divided into three major groups: linear responses, threshold responses, and tipping point responses (Scheffer et al. 2001) (Fig. 1). If a response function is linear, incremental changes in environmental factors will affect the system in proportional and predictable ways (Fig 1a). In linearly responding systems, reducing stressors can recover the system to its former state in the same way that a stretched rubber band will return to its initial shape once pulling is stopped.

In contrast, nonlinearly responding systems can change disproportionally to small changes in stressors with little or no warning. Threshold responses are a type of nonlinear response characterised by a sharp -but still reversible- change in system behaviour once a threshold stressor value is surpassed (Fig. 1b). Tipping point responses go one step further: they occur when a small change in the environmental stressor triggers a dramatic shift into an alternative state that is difficult to reverse (Fig. 1c). This means that a system that has shifted into an alternative state will not return to its previous state, even if conditions revert to pretransition levels. This phenomenon is known as 'hysteresis' and encapsulates the idea that the pathway to system degradation may not be the same as the pathway to restoration (Scheffer et 95 al. 2001). 


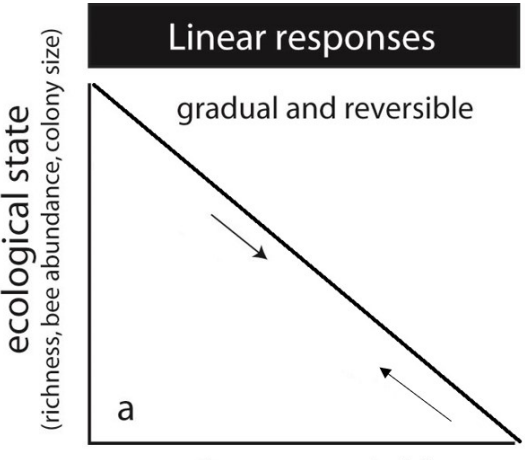

environmental driver

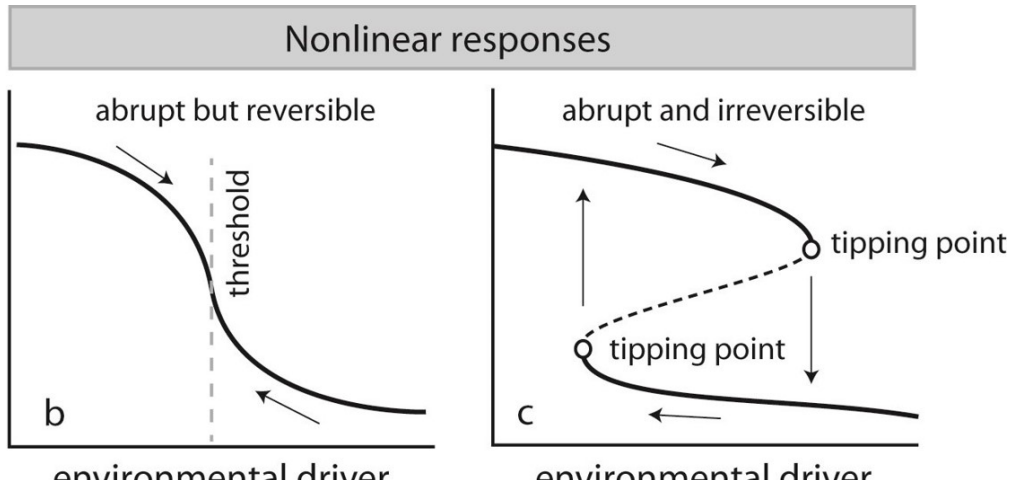

environmental driver

98 Figure 1 Type of responses in ecological systems due to a change in an environmental driver.

99 a) Linear decline. b) Nonlinear change of ecological state after the system passes a threshold.

100 c) Tipping point nonlinear response. At the tipping point, ecological systems shift from one

101 state (upper branch) to the alternative state (lower branch). Black solid lines indicate stable

102 equilibrium states, whereas black dotted lines represent the unstable threshold that separates

103 the basins of attraction of different stable states. The open circles indicate the tipping points

104 at which transitions between alternative states occur. (adapted from Scheffer et al (2001)).

In this paper, we synthesize theoretical and empirical evidence for nonlinear responses in pollination systems with emphasis on thresholds and tipping points. We conceptualise a pollination system as a hierarchical set of nested systems ('a system of systems') where pollinator-plant communities are made up of diverse and interacting pollinator and plant populations, which are, in turn, made up of interacting individuals that can adapt and exchange information. In the particular case of social insects, such as bumble

112 bees, stingless bees and honeybees, an extra level of organisation is added as individuals are

113 also organised into colonies, and colonies may interact with other colonies within a

114 population. At each level of organisation there are a separate set of dynamics that create

115 feedbacks across levels and result in emergent behaviours that cannot be anticipated if each

116 part of the system is considered in isolation (Levin 1998). In our framework, we explicitly

117 consider the potential that impacts on one level of the system can cascade through to other 118 levels.

119 Our first goal was to identify empirical and theoretical examples of thresholds and

120 tipping point responses at each level of the pollination system. Next, we explored the

121 potential for threshold responses to propagate across levels of the pollination system, arguing

122 that we need to explicitly consider their interactions and cascading effects. We conclude by

123 highlighting several open research questions that could improve our understanding of the

124 management and conservation implications of threshold and tipping point responses in

125 pollination systems. 

search

129 Evidence for strong nonlinear effects in pollination systems is likely scattered across many different literatures. We thus used a broad search strategy to capture the current state of evidence. We conducted searches of Web of Science using key words that describe strong nonlinear responses ("phase transition" or "bifurcation" or "tipping point" or "critical transition") and at least one of the following additional terms: "pollinator", "pollination", "Hymenoptera" (bees, ants, wasps and sawflies), "Diptera" (true flies), "Lepidoptera" (moths and butterflies) and "Coleoptera" (beetles). We chose the search terms Coleoptera, Diptera, Lepidoptera and Hymenoptera as we wanted to find papers that dealt with aspects of pollinator life history or physiology that could be missed with the "pollination" and "pollinator" search terms. We choose these four orders in particular as they are responsible for the majority of insect pollination (Rader et al 2016). In addition, we reviewed references obtained from the citation list of each paper. We also used knowledge of our respective fields to find additional examples that were not discovered using our search terms.

\section{Empirical evidence for tipping points and thresholds in pollinations systems} colony level (Bryden et al 2013), and no empirical examples at the population or community level. Although several studies investigated mechanisms that could plausibly lead to strong nonlinear declines (e.g Whitehorn et al 2009; Perry et al 2015), they did not collect the time series data and/or do the analyses necessary to confirm thresholds or tipping points. Since a wide variety of terminology is used to describe nonlinear stress responses, we cannot rule out the possibility that empirical examples exist but were not discovered by our search terms. While we did not find strong experimental or observational evidence for tipping point or threshold stress responses, we did discover evidence from theoretical studies. We argue that the 'ingredients' needed for nonlinear responses, such as strong positive feedback mechanisms (see Glossary), exist at each level of the pollination system. In the sections below, we discuss the theoretical knowledge base for threshold responses at the colony, population and community level.

\section{Threshold responses at the colony level: collapse of a pollinator society}

Despite the fact that the majority of bee species are solitary, social species such as honeybees, stingless bees and bumble bees are often crucial pollinators in managed and natural ecosystems (eg. Olesen et al. 2007; Geslin et al 2016). Social bee species are sometimes considered more resilient to environmental stressors than solitary species due to their 'superorganism resilience', where the large number of non-reproductive workers acts as a buffer against stress (Straub et al. 2015). If an individual bee dies, another rapidly takes her place; indeed, in several species there are exquisite physiological and/or behavioural 
166 mechanisms that allow individuals to alter their developmental trajectory to replace lost nest

167 mates (Robinson 1992). However, it is becoming increasingly clear that social bees may,

168 under certain conditions, suffer from colony collapses due to positive feedback mechanisms

169 within the colony (Bryden et al. 2013, Perry et al. 2015, Myerscough et al. 2017).

170 One of the clearest examples of a positive feedback leading to colony collapse comes

171 from the European honeybee (Apis mellifera). In honeybees, the task a worker bee performs

172 is dictated, in part, by her age. Younger workers typically perform in-nest tasks, while the

173 oldest workers function as foragers (Huang and Robinson 1996). Up to a critical mortality

174 rate, colonies can maintain a stable population size by replacing lost workers with sub-

175 optimally performing precocious foragers (bees that accelerate their development to become

176 foragers earlier than usual) (Khoury et al. 2011, Barron 2015, Perry et al. 2015, Myerscough

177 et al. 2017). However, precocious foragers are not as proficient as normal-aged foragers and

178 thus suffer from a higher mortality rate. Once the threshold mortality rate is surpassed, the

179 colony population declines precipitously as the workforce becomes increasingly young and

180 ineffective. This leads to a subsequent decline in food levels, which in turn stimulates the

181 recruitment of even younger bees into the foraging force (Perry et al. 2015). This death spiral

182 continues until the colony's adult workforce is depopulated and the colony ceases to function

183 (Fig. 2a). While compelling modelling strongly suggests the existence of tipping points or

184 thresholds in stressed honeybee colonies, strong empirical verification is lacking.

185 Models suggest that a high rate of forager loss (upwards of $30 \%$ of foragers dying per

186 day) would be needed to push honeybee colonies over the threshold (Perry et al. 2015). While

187 this is an extreme mortality rate (more than double the background mortality rate), it is not

188 unreasonable, especially when we consider that bees (and other central-place foragers) are

189 thought to be particularly sensitive to stressors that impair their cognitive function (Klein et

190 al. 2017). Cognitively impaired bees suffer high mortality because they cannot effectively

191 navigate, orient, communicate and/or learn. A wide range of stressors are known to impact

192 the cognitive function of bees including pesticides, heavy metals, parasites and pathogens,

193 malnutrition and low temperatures during development (Tautz et al. 2003, Gegear et al. 2006,

194 Jin et al. 2015, Burden et al. 2016, Klein et al. 2017). At least one study has found

195 experimental evidence that cognitive impairment alone is enough to trigger colony collapse in

196 bumblebees, where chronic exposure to sub-lethal doses of neonicotinoid insecticides

197 resulted in colony collapse when the number of cognitively impaired bees exceeded a critical 

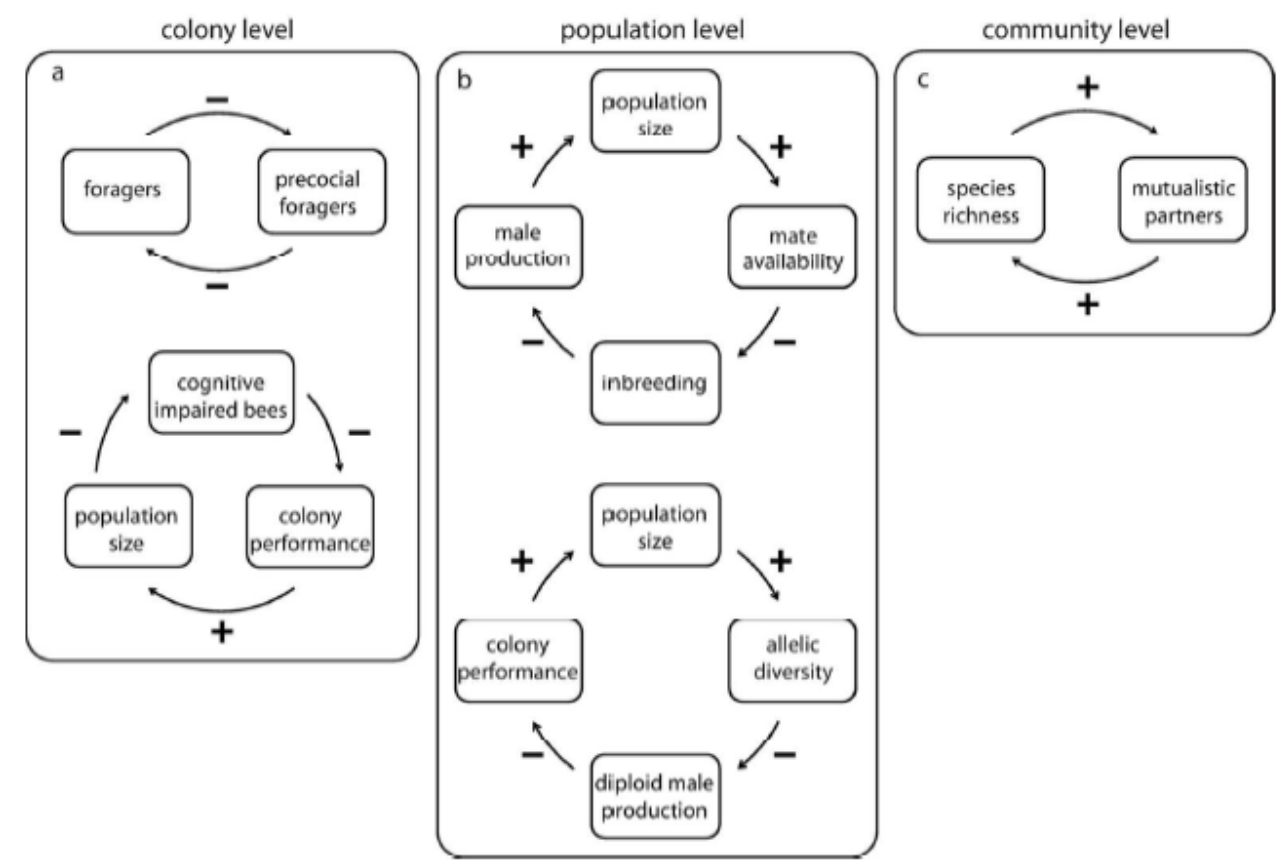

199

200

201

202

203

204

205

206

207

208

209

210

211

212

213

214

215

216

217

218

219

220

221

222

Figure 2. Known and suggested positive feedbacks that can possibly cause threshold and tipping point responses in pollination systems. Boxes contain system components, while arrows indicate interactions between components. Minus signs depict negative relationships between components, while plus signs indicate positive relationships. The product of the signs along the direction of the arrows results in a positive feedback process that may constitute a mechanism for causing threshold behaviour at each level of the pollination system.

\section{Threshold responses at the population level: Allee effects and extinction}

\section{vortices in pollinators}

In 1930, Allee observed that many species suffer from low or negative population growth

when their population size is small (Allee et al. 1949, Courchamp et al. 1999). Populations of small size would then suffer 'Allee effects' which drive them abruptly to extinction. At least

six mechanisms underlying Allee effects have been identified so far, including mate

limitation, cooperative defence, predator satiation, cooperative feeding, dispersal, and habitat alteration (Kramer et al. 2009). Most of these mechanisms could affect the population dynamics of pollinators. Butterflies, for example, may experience Allee effects due to mate limitation because individuals become reproductively active at different times of the year (Calabrese and Fagan 2004). At low population densities, more individuals find themselves reproductively isolated thus reducing the population growth rate and further decreasing the mating probability of individuals, ultimately leading to a spiral toward extinction. 
It is also possible for a population to be subject to multiple simultaneous Allee effects (Berec et al 2007). It is easy to imagine situations where an environmental driver (eg. insecticide use) causes an increase in mortality rate which then sets off an extinction spiral as Allee effects become more pronounced. Although the prevalence and magnitude of Allee effects amongst pollinators is unknown, it seems likely that they could increase the risk of extinction in pollinator populations at low densities.

In addition to Allee effects, a nonlinear shift to extinction at low densities can be enhanced by the combined effects of genetic diversity loss, environmental stochasticity, and demographic stochasticity. These elements create an 'extinction vortex' (Fig. 2b) that can cause accelerating decline leading to extinction (Gilpin 1986). Extinction vortices have been implicated in the extinction of ENREF 1810 vertebrate species (Fagan and Holmes 2006).

Pollinating insects in the order Hymenoptera may be at risk of a type of extinction vortex caused by their haplo-diploid sex-determination mechanism. In hymenopterans, sex is determined by the fertilization status of the egg; males are haploid and result from unfertilised eggs while females result from fertilized eggs and are diploid. In single locus complementary sex determination (sl-csd) (widespread in the Hymenoptera and believed to be ancestral), sex depends on the allelic composition of a single gene known as the csd (Van Wilgenburg et al. 2006). If a fertilized egg is heterozygous at the csd, it will develop into a normal diploid female (Hedrick et al. 2006). However, if the offspring is homozygous at the csd, the result is an inviable or sterile diploid male (Hedrick et al. 2006). A female that mates with a male with whom she shares one of her sex determination alleles will produce $50 \%$ inviable diploid males.

The production of diploid males results in mate limitation for fertile females, leading to decreased population size. If the population size continues to decline, genetic diversity at the csd also declines (Fig. 2b). As a consequence, the probability that a female will mate with a 'matched male' increases, thereby increasing the frequency of diploid males (Chapman and Bourke 2001). The resulting positive feedback loop, termed 'the diploid male vortex', ultimately drives the population to extinction at rates more than an order of magnitude higher than that caused by other forms of inbreeding depression (Zayed and Packer 2005).

Studies of inbred bumblebee colonies found that increased diploid male production was associated with a slower colony growth rate, shorter survival time and decreased offspring production suggesting the presence of an incipient diploid male vortex (Whitehorn et al. 2009). Darvill et al. (2012) found that the presence of infertile triploid male bumble bees (the product of a mating between a diploid male and a normal female) was negatively correlated with patch size, suggesting that environmental degradation caused an increase in triploid males as predicted by the diploid male vortex.

The risk of entering a diploid male vortex is influenced by several parameters. Modelling suggests that the number of diploid males in a population is driven more strongly 
by male flight radius than by the number of sexual alleles (Faria et al. 2016). The impact of male flight radius on allelic diversity is more pronounced in small patches than in larger patches (Faria et al. 2016). These results suggest that populations of smaller hymenopterans, which tend to have smaller flight ranges (Greenleaf et al. 2007), living in fragmented environments may be at increased risk of the diploid male vortex. The risk of diploid male vortices is exacerbated in social hymenopterans such as honeybees, stingless bees and bumble bees because only one or a few individuals within the colony actively engage in reproduction. As a result, effective population size can be orders of magnitude lower than the number of individuals observed in the field (Chapman and Bourke 2001).

Despite the dire implications of the diploid male vortex, some studies suggest that inbred populations can escape extinction. For example, Boff et al. (2014) found no relationship between island isolation and diploid male production in an orchid bee species even though genetic diversity was lower on islands. Similarly, Elias et al (2010) found no evidence for extinction proneness in parasitoid wasp populations containing high numbers of diploid males. Despite a massive population bottleneck, invasive Apis cerana honeybees apparently avoided the diploid male vortex because balancing selection prevented the loss of rare csd alleles, thus restoring high levels of heterozygosity (Gloag et al. 2016).

A modelling study by Hein et al. (2009) suggested that in order for the diploid male vortex to start, four conditions needed to be met: (i) extreme fragmentation (i.e., populations are small and isolated), (ii) fixed sex ratio, (iii) low reproductive rate, and (iv) an inability on the part of females to detect the genotype of potential mates. Relaxing any of these conditions allows the population to escape the diploid male vortex. While these theoretical results may reduce the set of conditions under which a diploid male vortex can develop, the combination of even a weak diploid male vortex with unknown (so far) Allee effects might still lead to nonlinear responses to extinction in pollinator populations.

Threshold responses at the community level: extinction cascades and the collapse of pollinator communities

290 Pollinator communities are made up of interacting flowering plants and their pollinators whose interactions form ecological networks. Ecological networks provide a powerful conceptual tool for understanding the influence of environmental change on communities (Pascual 2006). Such influence can take place due to changes in species composition, due to changes in realised species interactions, or to changes in the co-evolutionary processes that shape species interactions (Tylianakis and Morris 2017).

A characteristic property of pollination networks is their 'nested' structure, which means that specialist species tend to interact with a core composed of their most generalist partners (Bascompte et al. 2003, Bascompte and Jordano 2007). There is a substantial amount 
of theoretical literature that aims to understand the role of this nested topology on the stability and maintenance of biodiversity in mutualistic communities such a plant-pollinator networks.

Nested plant-pollinator communities have been shown to sustain high biodiversity (Bastolla et al 2009) and to remain stable to random disturbances (Grilli et al 2016) when compared to their random counterparts. On the other hand, nested pollination networks are more prone to nonlinear community-wide collapses compared to random networks without a nested structure (Lever et al. 2014). The increased susceptibility to collapse occurs because in highly nested communities a strong positive feedback can develop between pollinators that interact with the same plant. Simply put, in a nested pollination network there are more pollinator species that interact with the same plant species. These multiple shared interactions translate into a higher number of pollinator visitors for the plant. Since the mutualistic benefit to the pollinators is proportional to plant biomass, pollinators can survive high levels of stress because they are benefiting from indirect positive effects from their nested pollinator partners. The positive indirect effect sustains the existence of the pollinators up to a threshold stress level. Once the stress threshold is surpassed the indirect effects are no longer sufficient at which point all nested partners collapse to extinction.

Nonlinear species loss is expected to occur predominantly in communities where plants depend strongly on their pollinators and vice versa (obligate mutualism) (Lever et al 2014, Dakos and Bascompte 2014). The nonlinear response is usually a sequence of cascading tipping point events where subsets of species go extinct from the community. In addition to nestedness, higher degree of connectance (i.e. the realised number of interactions over the total possible number in a network) results in a higher probability that these tipping point events become synchronised; this synchronicity ultimately leads to a single strong response affecting most of the community (Lever et al 2014).

However, the pattern of community extinction in pollination systems under stress is not necessarily nonlinear. Species extinctions in plant-pollinator communities may follow a sequential pattern occasionally triggering cascading secondary extinctions, but where the overall pattern of species loss will appear gradual rather than abrupt. Whether or not the pattern of extinction is linear or nonlinear is influenced by each species' tolerance to extinction which in turn depends on the species' number of interactions and on its contribution to network nestedness (Saavedra et al 2013). Memmott et al. (2004) found that removing pollinators from the most specialist to the least specialist species led to nonlinear response only after the majority of pollinators had been lost. However, when pollinators were removed in the reverse order (least specialist to most specialist) plant species went extinct more rapidly but in a linear manner.

Which extinction pattern (least specialist to most specialist or vice versa) is more likely to occur empirically? Aizen et al (2012) found evidence that specialist pollinator species with a small number of interactions were more likely to disappear from isolated hills in Argentina. Using a 120 year-long dataset, Burkle et al (2013) found that specialist 
338 pollinators were more likely to be extirpated, even when their host plants were still present.

339 These empirical findings suggest that specialist pollinators are more likely to go extinct than

340 generalists.

341 Other empirical studies show that invasive species can also alter the structure of

342 pollination networks (Aizen et al 2008) thus altering the probability of nonlinear collapses.

343 For instance, exotic plants are often generalists, attracting a large number of pollinator

344 species (Aizen et al 2008, Stouffer et al 2014) which can cause invaded communities to

345 become significantly more nested (Bartomeus et al 2008). If such patterns are generally true,

346 the loss of specialist species or the invasion of generalist species may lead to strongly

347 connected or nested networks that will be more likely to suffer strong, sudden synchronised

348 collapses.

349 The prediction that networks may become prone to synchronised collapses neglects

350 the potential buffering effect of 'interaction rewiring', which has been empirically shown to

351 contribute to maintaining a constant network structure (Petanidou et al 2008, Timoteo et al

352 2016). Interaction rewiring refers to a pollinator's ability to switch to alternative, less-

353 preferred flowers if a preferred flower is unavailable; this behaviour might prevent a

354 pollinator population from collapsing long enough for a preferred plant species to

355 recolonise the site. Removal experiments conducted on simulated Mauritian pollination

356 networks found that network stability can be maintained if species 're-wire' themselves by

357 shifting to new plants when their preferred species becomes extinct (Kaiser-Bunbury et al.

358 2010).

359 More generally, adaptive foraging by pollinators can increase community persistence

360 and robustness to species loss (Valdovinos et al. 2013). The potential of adaptive responses to

361 stabilise communities will depend partly on the amount and conservation of trait variation

362 within populations that could counteract phenological mismatches between plants and

363 pollinators (Revilla et al. 2015) or the sensitivity of specific species to chemical pollution

364 (reviewed in Leonard and Hochuli 2016). In addition, the effect of network rewiring via

365 adaptive foraging will depend on the temporal and spatial variability of interactions

366 (Tylianakis and Morris 2017). Climate-induced phenological shifts in plant-pollinator

367 communities (Rafferty et al 2013) can leave plants and pollinators without interactions even

368 if the phenological shifts are small (Memmott et al 2007) and could potentially increase the

369 risk of a community collapse. So far there is insufficient empirical evidence and an

370 incomplete theoretical understanding of the extent to which behavioural changes, flows

371 between meta-communities, and trait variation act in concert to minimize the risk of

372 threshold responses in pollination networks.

374 Open research questions 
375 Our synthesis of theoretical and empirical evidence for nonlinear effects at different levels of

376 the pollination system revealed several missing links and open questions. Below we address several areas where further knowledge could improve our understanding and management of thresholds and tipping points in pollination systems.

\section{How prevalent are threshold and tipping point responses?}

The existence of positive feedback loops at all levels of the pollination system lead us to the prediction that nonlinear stress responses should be common. Yet our literature search failed to find either experimental or observational evidence for nonlinear phenomenon at the population or community level and only one clear example from the colony level. Why is there a mismatch between prediction and experimental results? One possibility is that real pollination systems have a number of resilience mechanisms (discussed below) that allow for greater -than-expected tolerance to stressors. Nonlinear effects are also, by their very nature, difficult to observe until collapse is already underway. It is also possible that more examples of nonlinear responses exist but were not captured by our search terms. We suspect that researchers may not always frame their results in terms of thresholds and tipping points, even when studying systems that display these dynamics. Thus we suggest that future work focus on characterising the shape of stress responses at all levels of the pollination system; this would allow us to determine the actual prevalence of nonlinear effects.

Are there mechanisms that safeguard the stability of pollinator systems and reduce the risk of threshold and tipping point responses?

It is possible that resilience mechanisms (such as network rewiring, flexible caste determination, demographic rescue from nearby populations, conservation of genetic variation) are sufficient to delay or prevent the kind of nonlinear responses we describe throughout this manuscript. However the extent and nature of these mechanisms is still

401 largely unknown. With further research, it may be possible to harness the stabilising ability

402 of resilience mechanisms to buffer systems that are close to tipping points. In the case of

403 urban and agricultural environments, this could take the form of selective planting of key

404 plant species, or the provision of nesting habitat targeted at pollinator species known to play

405 key roles in maintaining network resilience. On the conservation side, a thorough

406 understanding of resilience mechanisms may allow us to prioritise the conservation of particularly important plant or pollinator species. At the colony level, understanding resilience mechanisms could lead to new interventions for preventing colony collapse in 
413 A key step towards safeguarding pollination systems is to identify which systems (if any)

414 have a high risk of threshold or tipping point responses. This will require studies aimed at

415 identifying key stressors, positive feedback mechanisms, nonlinear responses in observational

416 data, and, if possible, experiments for establishing the existence of thresholds (Scheffer and

417 Carpenter 2003). Here, we have tentatively identified places where there is potential for

418 threshold or tipping point responses (Fig. 2), but further work is needed to confirm and

419 expand our list. This is no easy task as experimental evidence is difficult to gather (Schröder

420 et al. 2005). So far, studies using space-for-time substitution document gentle rather than

421 nonlinear decreases in abundance of bee populations along disturbance gradients (Winfree et

422 al. 2009). Nonetheless, such observed response patterns are few, remain difficult to obtain,

423 and do not preclude the potential for threshold or tipping point responses. A meta-analysis of

424 studies focused on long term population trends in pollinating species might be helpful in

425 determining whether population declines tend to be linear or nonlinear. Unfortunately, longer

426 term studies at the required resolution remain few and far between.

427 At the colony level, experiments that carefully apply varying stressors to bee colonies

428 could be used to determine the shape of the colonies' response function. It would be

429 particularly interesting to determine if different stressors result in different types of decline

430 (eg. threshold, tipping point, linear). The data needed to address these questions might

431 already exist within data sets collected from the increasing number of sensor-enabled

432 honeybee hives. In addition, modelling of bee colony demography and behaviour can be used

433 to narrow down the list of potential stressors (and their interactions) that are likely to cause

434 nonlinear declines; once candidate stressors have been identified in silico they can then be

435 tested in the field (Henry et al 2017).

436 Systems with tipping points can appear gradual in their responses to stress due to time

437 lags (Hughes et al. 2013). Slowly responding populations under rapid environmental change,

438 assisted by subsidies between metacommunities, can create temporal and spatial

439 heterogeneities that may mask the nonlinear response of a bistable system. Indeed, threshold

440 responses at the colony, population and community level will most likely be identified after

441 terminal collapse processes are underway because timescales of ecological responses often do

442 not match monitoring timescales. In addition, traditional census techniques (eg. pollinator

443 sampling) which do not take into account the potential for nonlinear responses might

444 overestimate the true health of pollination systems. What is needed is a reliable way to

445 identify the conditions that can increase the potential fragility of pollination systems (see

446 (Foley et al. 2015, Hunsicker et al. 2016). Research that combines modelling and field

447 experiments can help achieve this goal.

449 Could interacting threshold and tipping point responses lead to cascading 450 failures across scales in a pollination system? 
451 Thus far we have described real and potential threshold or tipping point responses at colony,

452 population and community levels in isolation (Fig. 2). However, levels of the pollination

453 system can interact with one another, potentially accelerating global collapse (Fig. 3). It is

454 well-recognised that different environmental stressors can interact with one another leading

455 to synergistic effects on pollinator decline (eg. Vanbergen 2013, Goulson et al. 2015,

456 Gonzalez-Varo et al 2013). What is less well-studied is the potential for interactions between

457 different levels of the pollination system to cause cascading effects that could increase the

458 risk of pollination system collapse. Consider, for instance, that exposure to sublethal doses of

459 insecticides could result in the collapse of wild bumblebee colonies if a threshold number of

460 bees experience cognitive impairment. A local increase in the rate of colony failure could

461 reduce population-level genetic diversity, initiating a diploid male vortex. This would trigger

462 a decrease in the number of social bees, which are often important, highly connected

463 components of the pollination network (Olesen et al. 2007). The loss of social bees could in

464 turn initiate a series of plant extinctions, resulting in a cascade of secondary extinctions and,

465 ultimately, community collapse.

466 How likely is this scenario? Although there are mechanisms that can buffer responses

467 in the pollination system (like the ones described in the previous section), the extent to which

468 resilience mechanisms can prevent collapse in the face of rapidly increasing environmental

469 change remains unknown. Thus, research is needed to understand how different nonlinear

470 responses of the pollination system at different scales might interact to cause global systemic

471 collapses (Fig. 3). 


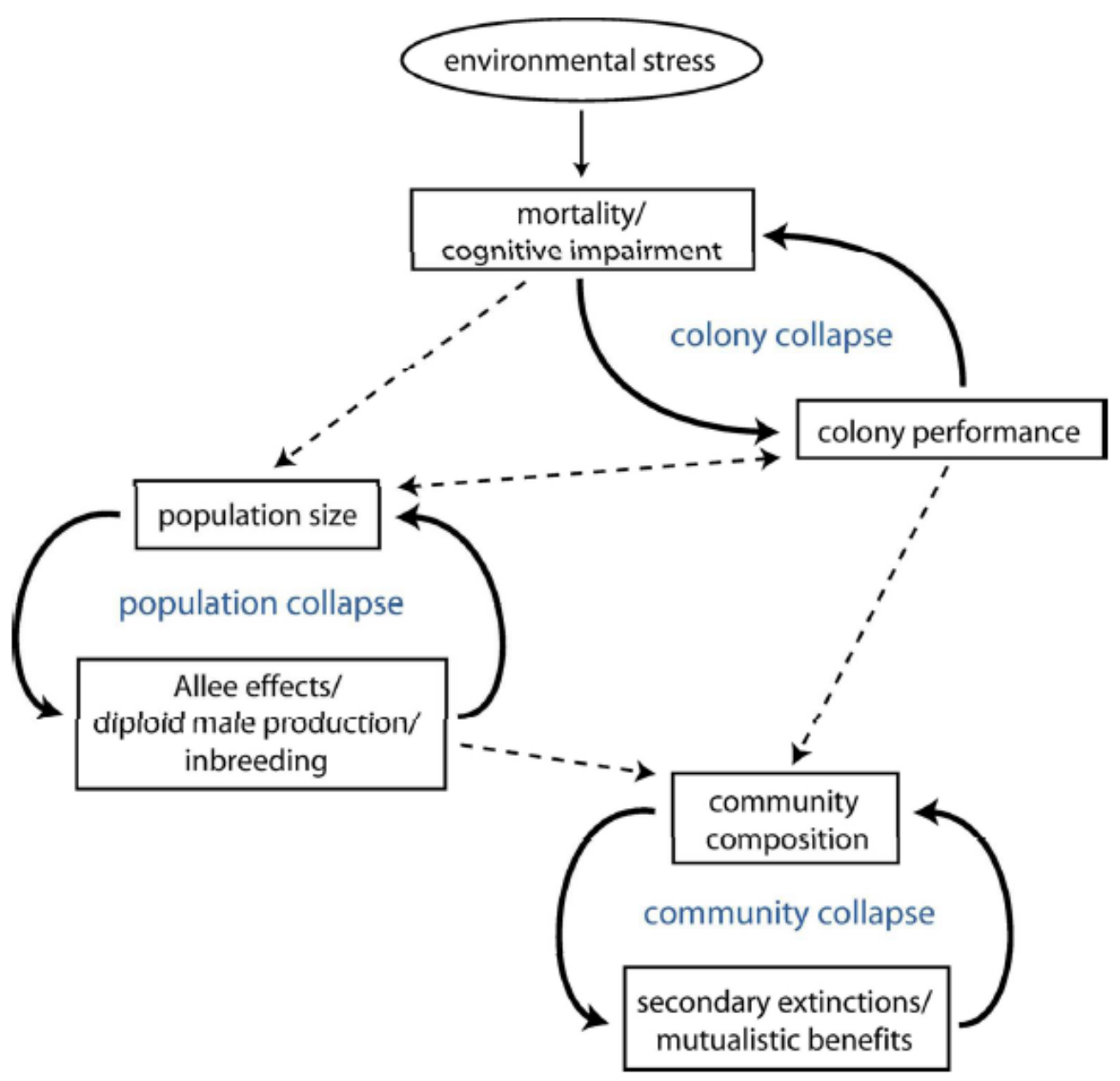

482 Figure 3. Potential interconnections between positive feedbacks across different levels of organisation in the pollination system. Potential pathways are depicted via which environmental stresses can create a series of cascades that could increase the risk of collapse in a pollination system. Straight dotted arrows indicate interactions between different components. The solid curved arrows depict positive feedback processes. Allee effects, diploid male effects, and inbreeding are grouped together for ease of representation. Colony collapse occurs at the colony level, extinction vortices occur at the population level, and extinction cascades occur at the community level. 
491 Which pollinator species are most susceptible to threshold and tipping point responses at the population level?

493 Ideally, species or populations at risk of non-linear declines would be identified as potential targets for monitoring and conservation efforts. The hymenopteran sex determination system and the fact that hymenopterans are central place foragers could potentially make them more vulnerable than other insect groups to non-linear declines. However, we currently have little knowledge on how common extinction vortices and diploid male vortices are in nature nor do we know whether there are strong Allee effects in non-hymenopteran pollinators. At the colony level, experimental evidence for non-linear declines in colony health comes only from bumble bees; examples from other eusocial bee species are lacking although there is strong anecdotal evidence of rapid, nonlinear collapse in honeybees. Future research should determine if non-linear decline is a common feature of colony failures or if it is associated with particular stressors.

Another unresolved question is the extent to which eusocial species are more sensitive to environmental change than are solitary species. The potential fragility of social species is suggested by several recent analyses which found that sociality in bees is associated with a lack of resilience to land use changes (Williams et al. 2010, De Palma et al. 2015 although see Bartomeus et al 2013 for a counter example).

\section{Can we develop indicators for detecting impending pollinator collapse?}

A possible method for assessing the risk of nonlinear responses in pollination systems comes from a set of methods called resilience indicators. Resilience indicators are based on statistical properties in the dynamics of a system that are symptomatic of an impending tipping point (Scheffer et al 2009). The most prominent of these indicators are a rise in variance and autocorrelation, both of which are consequences of the slow response capacity of a system close to a tipping point (Wissel 1984). General frameworks for the application and use of these indicators are well-developed (Dakos et al. 2012), and they have been increasingly tested in lab and field experiments (Carpenter et al. 2011, Dai et al. 2012, Veraart et al. 2012, Gsell et al. 2016). In fact, these indicators have been shown to be generic for a range of ecological responses which makes them universal signals of degradation and loss of ecological resilience (Kéfi et al. 2013). Although so far there is no empirical testing of early warning indicators in pollination systems, these indicators have been theoretically shown to provide warning of impeding collapse both at the species and community level in simulations of empirically described plant pollinator networks under increasing environmental stress (Dakos and Bascompte 2014). At the colony level, recent advances in high frequency monitoring via sensor arrays inside managed bee colonies could be used to collect a large amount of data about colony health and performance. These metrics, alone or 
528 in combination, could serve as response variables, which could be analysed for early warning

529 indicators

530 It may be particularly difficult to adapt generic early warning indicators to the

531 community and population levels of pollination systems because they require long and highly

532 resolved records of population abundances; while such data may exist for some taxa in some

533 places, it is difficult to collect and unavailable for the majority of pollinator species.

534 Theoretical work suggests that it may be possible to decrease this complexity by reducing

535 multispecies community dynamics to aggregated dynamics of plant and pollinators functional

536 groups (Jiang et al 2018). It remains, however, a challenge to achieve this simplification as it

537 requires information on both the structure and the intensity of mutualistic interactions

538 between plants and their pollinators.

$539 \quad$ Alternatively, response variables such as crop yield, fruit quality or farmer profit

540 could be attractive targets for analysis with early warning indicators because they are

541 relatively easy to collect and are likely linked to the functioning of the pollination system.

542 Theoretically, it has been shown that changes in variance and autocorrelation of profit made

543 by the exploitation of a harvested resource (e.g. fisheries) could better reflect the risk of

544 collapse compared to changes in the same indicators of the state of the system itself (Richter

545 and Dakos 2015). However, the use of yield, profit or quality might be complicated in

546 agricultural systems because of human management. For example, growers might

547 compensate for decreased crop yields due to the loss of wild pollinators by renting honeybee

548 colonies; this would mask early warning signals in crop yield data. Indeed, it has been

549 suggested that human adaptive management tends to keep agricultural systems close to their

550 tipping points (Yletyinen et al 2019), which might dampen the effectiveness of early warning

551 indicators (Bauch et al 2016). For this reason, a combination of ecological and

552 socioeconomic response variables might be more useful (Yletyinen et al 2019). For instance,

553 in the case of agricultural pollination, monitoring could include the amount of money growers

554 allocate to renting honeybee hives as well as floral visitation rate and/or pollinator species

555 richness. Progress toward identifying suitable response variables will require simulation

556 studies to explore scenarios of how the combination of abundance dynamics, structural

557 community properties and human responses could create synthetic indicators for the risk of

558 abrupt community responses that could be tested in the real-world.

\section{How do we manage threshold responses in pollination systems?}

561 Insight into how to best manage threshold effects in pollination systems could come from the 562 experiences of managers in other ecological systems. In their analysis of 51 case studies,

563 (Kelly et al. 2015) found that ecological systems with strong nonlinear responses could be

564 best managed when the systems were relatively small (so that inputs and outputs could be 565 more closely controlled), when routine monitoring programs were in place, and when systems 566 were specifically managed with nonlinearity, thresholds and tipping points in mind. 
Long term pollinator monitoring programs are urgently needed especially in poorly

568 studied regions of the world such as Australia, Africa and Asia. A precautionary attitude

569 should be taken when setting conservation targets for pollination systems, since declines may

570 happen suddenly and, in the case of bistable systems, may be irreversible. Primary industries

571 such as agricultural systems may be at particular risk for tipping points and the economic

572 impacts of such collapse could be very high (Yletyinen et al 2019). Thus, we suggest there is

573 an urgent need for research into nonlinear responses in pollination-dependent crops.

$574 \quad$ The finding that management outcomes were most successful when managers

575 specifically managed with thresholds and critical transitions in mind is promising; it is our

576 hope that the present manuscript will encourage managers and researchers to think about

577 potential threshold responses within pollination systems.

578

579 Concluding remarks

580 Tipping points and thresholds are, by their nature, difficult to detect until they have been

581 passed. As such, the lack of direct field evidence for nonlinear effects at the community and

582 population level should not lead to complacency. While pollination systems can be resilient

583 against environmental change, collapse of these systems would be economically and

584 ecologically catastrophic. Since environmental change will almost certainly continue to

585 accelerate, understanding the mechanisms that can provoke collapses and developing

586 techniques to prevent them may be key to safeguarding our pollination systems.

\section{Acknowledgements}

592 The authors would like to thank Ignacio Bartomeus for his valuable comments and

593 suggestions. We would also like to thank Johanna Yletyinen for kindly providing an early

594 copy of her manuscript. Neither author declares a conflict of interest. 


\begin{tabular}{|c|c|}
\hline Allee effect & $\begin{array}{l}\text { Allee effects occur when populations suffer from low or negative } \\
\text { population growth when their population size is small. }\end{array}$ \\
\hline $\begin{array}{l}\text { Alternative stable } \\
\text { states }\end{array}$ & $\begin{array}{l}\text { Different states of a system that can occur under the same external } \\
\text { conditions. }\end{array}$ \\
\hline Bistability & $\begin{array}{l}\text { The presence of two alternative stable states under the same } \\
\text { conditions. }\end{array}$ \\
\hline Extinction vortex & $\begin{array}{l}\text { A self-reinforcing process that drives population size downward to } \\
\text { extinction. }\end{array}$ \\
\hline Hysteresis & $\begin{array}{l}\text { The lack of reversibility in bistable systems; hysteresis refers to the } \\
\text { phenomenon where the pathway to system degradation may not be } \\
\text { the same as the pathway to restoration. }\end{array}$ \\
\hline Threshold response & $\begin{array}{l}\text { A strong nonlinear response of a system to small changes in } \\
\text { environmental conditions or stressors }\end{array}$ \\
\hline Pollination system & $\begin{array}{l}\text { Community composed of interacting pollinators (animals) and } \\
\text { plants. }\end{array}$ \\
\hline Positive feedback & $\begin{array}{l}\text { A self-amplifying process between two or more system } \\
\text { components. }\end{array}$ \\
\hline Resilience indicators & $\begin{array}{l}\text { Indicators of increasing instability in system dynamics that are used } \\
\text { to detect proximity to tipping points (also referred to as early- } \\
\text { warning signals). }\end{array}$ \\
\hline Tipping point & $\begin{array}{l}\text { A point where a runaway process (usually due to a positive } \\
\text { feedback) pushes a system to flip into a different state. }\end{array}$ \\
\hline
\end{tabular}




\section{REFERENCES}

Aizen, M.A., Morales, C.L. and Morales, J.M., 2008. Invasive mutualists erode native pollination webs. PLoS biology, 6(2):31

600

Aizen, M. A. and L. D. Harder 2009. The global stock of domesticated honey bees is growing slower than agricultural demand for pollination. Current Biology 19(11): 915-918.

603

Aizen MA, Sabatino M, Tylianakis JM 2012. Specialization and rarity predict nonrandom loss of interactions from mutualist networks Science 335:1486-1489

606

Allee, W. C., O. Park, A. E. Emerson, T. Park, and K. P. Schmidt. 1949. Principles of animal ecology. WB Saundere Co. Ltd.

609

Arbetman, M. P., G. Gleiser, C. L. Morales, P. Williams, and M. A. Aizen. 2017. Global decline of bumblebees is phylogenetically structured and inversely related to species range size and pathogen incidence. Proc. R. Soc. B 284:20170204.

613

Bartomeus, I., Vilà, M. and Santamaría, L., 2008. Contrasting effects of invasive plants in plantpollinator networks. Oecologia 155(4): 761-770. biodiversity. Annu. Rev. Ecol. Evol. Syst. 38:567-593. animal mutualistic networks. Proceedings of the National Academy of Sciences 100:9383-9387. Berec L., Angulo E. \& Courchamp F. 2007 Multiple Allee effects and population management. 
630 Biesmeijer, J. C., Roberts, S. P., Reemer, M., Ohlemüller, R., Edwards, M., Peeters, T. Thomas, C

631 and Settele, J. 2006. Parallel declines in pollinators and insect-pollinated plants in Britain and the

$632 \quad$ Netherlands. Science, 313(5785): 351-354.

633

634 Bastolla U, Fortuna MA, Pascual-García A, Ferrera A, Luque B, Bascompte J. The architecture of 635 mutualistic networks minimizes competition and increases biodiversity. Nature. 2009

$636 \quad 458(7241): 1018-1021$

637

638

639

Bauch, C.T., Sigdel, R., Pharaon, J. and Anand, M., 2016. Early warning signals of regime shifts in coupled human-environment systems. Proceedings of the National Academy of Sciences,

641

Boff, S., A. Soro, R. J. Paxton, and I. Alves-dos-Santos. 2014. Island isolation reduces genetic diversity and connectivity but does not significantly elevate diploid male production in a neotropical orchid bee. Conservation genetics 15:1123-1135.

Brosi, B. J., and H. M. Briggs. 2013. Single pollinator species losses reduce floral fidelity and plant reproductive function. Proceedings of the National Academy of Sciences 110:13044-13048. Bryden, J., R. J. Gill, R. A. Mitton, N. E. Raine, and V. A. Jansen. 2013. Chronic sublethal stress causes bee colony failure. Ecology Letters 16:1463-1469. selenium disrupts associative conditioning and long-term memory recall in honey bees (Apis mellifera). Ecotoxicology and Environmental Safety 127:71-79. asynchrony and the Allee effect. The American Naturalist 164:25-37. 
Carvalheiro, L. G., W. E. Kunin, P. Keil, J. Aguirre Gutiérrez, W. N. Ellis, R. Fox, Q. Groom, S. homogenisation have slowed down for NW European pollinators and plants. Ecology letters 16(7): 870-878.

669

Chacoff, N. P., J. Resasco, and D. P. Vázquez. 2018. Interaction frequency, network position, and the temporal persistence of interactions in a plant-pollinator network. Ecology 99:21-28. of social insects. Ecology Letters 4:650-662.

Courchamp, F., T. Clutton-Brock, and B. Grenfell. 1999. Inverse density dependence and the

Dakos, V., and J. Bascompte. 2014. Critical slowing down as early warning for the onset of D. A. Seekell, and E. H. van Nes. 2012. Methods for detecting early warnings of critical transitions in time series illustrated using simulated ecological data. PLoS One 7:e41010. 
694 Newbold, and A. Purvis. 2015. Ecological traits affect the sensitivity of bees to land-use pressures in European agricultural landscapes. Journal of Applied Ecology 52:1567-1577. decreasing effective population size in a parasitoid with complementary sex determination and fertile diploid males. BMC Evolutionary Biology, 10(1), 366. doi:10.1186/1471-2148-10-366

Fagan, W. F., and E. Holmes. 2006. Quantifying the extinction vortex. Ecology Letters 9:51-60.

702

Faria, L. R. R., E. D. G. Soares, E. d. Carmo, and P. M. C. d. Oliveira. 2016. Diploid male dynamics under different numbers of sexual alleles and male dispersal abilities. Theory in Biosciences 135:111-119. removal: pollinators stay but some native plants miss their new friend. Biological Invasions $15: 2347-2358$. $2: 95$. mutualistic networks. Ecology Letters 9:281-286. mutalistic networks. Oikos 122:938-942. 
Garibaldi, L. A., et al. 2013. Wild pollinators enhance fruit set of crops regardless of honey bee abundance. Science 339(6127): 1608-1611.

Gegear, R. J., M. C. Otterstatter, and J. D. Thomson. 2006. Bumble-bee foragers infected by a gut parasite have an impaired ability to utilize floral information. Proceedings of the Royal Society of London B: Biological Sciences 273:1073-1078.

730

Gilpin, M. 1986. Minimum viable populations: Processes of extinction in ME Soulé editor. Conservation Biology: The Science of Scarcity and Diversity. Sinauer Associates, Sunderland, MA. invasive social insect overcomes genetic load at the sex locus. Nature Ecology \& Evolution $1: 0011$

Goldstein, J., and M. Zych. 2016. What if we lose a hub? Experimental testing of pollination network resilience to removal of keystone floral resources. Arthropod-Plant Interactions 10:263271. González-Varo, J.P., Biesmeijer, J.C., Bommarco, R., Potts, S.G., Schweiger, O., Smith, H.G., Steffan-Dewenter, I., Szentgyörgyi, H., Woyciechowski, M. and Vilà, M., 2013. Combined effects of global change pressures on animal-mediated pollination. Trends in Ecology \& Evolution, 28(9), pp.524-530.

Goulson, D., E. Nicholls, C. Botías, and E. L. Rotheray. 2015. Bee declines driven by combined stress from parasites, pesticides, and lack of flowers. Science 347:1255957.

Greenleaf, S. S., N. M. Williams, R. Winfree, and C. Kremen. 2007. Bee foraging ranges and their relationship to body size. Oecologia 153:589-596.

Gsell, A. S., U. Scharfenberger, D. Özkundakci, A. Walters, L.-A. Hansson, A. B. G. Janssen, P. Nõges, P. C. Reid, D. E. Schindler, E. Van Donk, V. Dakos, and R. Adrian. 2016. Evaluating 
early-warning indicators of critical transitions in natural aquatic ecosystems. Proceedings of the

Hedrick, P. W., J. Gadau, and R. E. Page. 2006. Genetic sex determination and extinction. Trends in Ecology \& Evolution 21:55-57.

Hein, S., H.-J. Poethke, and S. Dorn. 2009. What stops the 'diploid male vortex'? - A simulation study for species with single locus complementary sex determination. Ecological Modelling 220:1663-1669.

Henry, M., Becher, M.A., Osborne, J.L., Kennedy, P.J., Aupinel, P., Bretagnolle, V., Brun, F., Grimm, V., Horn, J. and Requier, F., 2017. Predictive systems models can help elucidate bee declines driven by multiple combined stressors. Apidologie: 48(3), pp.328-339.

Huang, Z.-Y., and G. E. Robinson. 1996. Regulation of honey bee division of labor by colony age demography. Behavioral Ecology and Sociobiology 39:147-158. dangerously on borrowed time during slow, unrecognized regime shifts. Trends in Ecology \& Evolution 28:149-155. Amrhein. 2016. Characterizing driver-response relationships in marine pelagic ecosystems for improved ocean management. Ecological Applications 26:651-663. 

of pollination networks to the loss of species and interactions: a quantitative approach incorporating pollinator behaviour. Ecology Letters 13:442-452.

Kéfi, S., V. Dakos, M. Scheffer, E. H. Van Nes, and M. Rietkerk. 2013. Early warning signals also precede non-catastrophic transitions. Oikos 122:641-648. Embracing thresholds for better environmental management. Philosophical Transactions of the Royal Society of London B: Biological Sciences 370:20130276.

Khoury, D. S., M. R. Myerscough, and A. B. Barron. 2011. A quantitative model of honey bee colony population dynamics. PLoS One 6:e18491.

Klein, A.-M., B. E. Vaissiere, J. H. Cane, I. Steffan-Dewenter, S. A. Cunningham, C. Kremen, and T. Tscharntke. 2007. Importance of pollinators in changing landscapes for world crops. Proceedings of the Royal Society B: Biological Sciences 274:303-313.

Klein, S., A. Cabirol, J.-M. Devaud, A. B. Barron, and M. Lihoreau. 2017. Why Bees Are So Vulnerable to Environmental Stressors. Trends in Ecology \& Evolution. effects. Population Ecology 51:341. biodiversity conservation. Trends in ecology \& evolution, 24(10), pp.564-571. 
815 Lever, J. J., E. H. Nes, M. Scheffer, and J. Bascompte. 2014. The sudden collapse of pollinator

816 communities. Ecology Letters 17:350-359.

817

818 Levin, S.A., 1998. Ecosystems and the biosphere as complex adaptive systems. Ecosystems,

$819 \quad 1(5), 431-436$

820

821 Majdandzic, A., L. A. Braunstein, C. Curme, I. Vodenska, S. Levy-Carciente, H. E. Stanley, and 822 S. Havlin. 2016. Multiple tipping points and optimal repairing in interacting networks. Nature $823 \quad$ communications 7 .

824

825

Manley, R., M. Boots, and L. Wilfert. 2015. Emerging viral disease risk to pollinating insects: ecological, evolutionary and anthropogenic factors. Journal of Applied Ecology 52:331-340. Memmott, J., N. M. Waser, and M. V. Price. 2004. Tolerance of pollination networks to species extinctions. Proceedings of the Royal Society of London B: Biological Sciences 271:2605-2611.

Memmott, J., Craze, P.G., Waser, N.M. and Price, M.V., 2007. Global warming and the disruption of plant-pollinator interactions. Ecology letters 10(8): 710-717. Using Mathematics to Solve the Problem of Honey Bee Colony Collapse. Pages 35-50 The Role and Importance of Mathematics in Innovation. Springer. networks. Proceedings of the National Academy of Sciences 104:19891-19896. animals? Oikos 120:321-326. 

invariance of network structure and implications for estimates of specialization. Ecology letters 11: 564-575.

855

Potts, S. G., Biesmeijer, J. C., Kremen, C., Neumann, P., Schweiger, O., \& Kunin, W. E. 2010. S.A., Mayfield, M.M., Arthur, A.D., Andersson, G.K. and Bommarco, R., 2016. Non-bee insects are important contributors to global crop pollination. Proceedings of the National Academy of overlap of interacting species in a changing climate: an assessment of available approaches. Ecology and evolution 3(9):3183-3193. 
879 promote feasibility over stability during the assembly of a pollinator community. Ecology and

$880 \quad$ evolution 6: 997-1007.

881

Saavedra S, Stouffer DB, Uzzi B, Bascompte J,. 2011. Strong contributors to network persistence

883 are the most vulnerable to extinction Nature 478:233- 236

884

885

Sánchez-Bayo, F., \& Wyckhuys, K. A. G. 2019. Worldwide decline of the entomofauna: A

886 review of its drivers. Biological Conservation, 232, 8-27.

887

888

Scheffer, M. 2009. Critical transitions in nature and society. Princeton University Press.

889

Scheffer, M., J. Bascompte, W. A. Brock, V. Brovkin, S. R. Carpenter, V. Dakos, H. Held, E. H. van Nes, M. Rietkerk, and G. Sugihara. 2009. Early-warning signals for critical transitions. Nature 461:53-59.

893 ecosystems. Nature 413:591-596. stable states: a review. Oikos 110:3-19. in Insect Science 12:109-112. 
911 Suweis, S., F. Simini, J. R. Banavar, and A. Maritan. 2013. Emergence of structural and

912 dynamical properties of ecological mutualistic networks. Nature 500:449-452.

913

914 Tautz, J., S. Maier, C. Groh, W. Rössler, and A. Brockmann. 2003. Behavioral performance in

915 adult honey bees is influenced by the temperature experienced during their pupal development.

916 Proceedings of the National Academy of Sciences 100:7343-7347.

917

918 Timóteo, S., Ramos, J.A., Vaughan, I.P. and Memmott, J., 2016. High resilience of seed dispersal 919 webs highlighted by the experimental removal of the dominant disperser. Current Biology 26(7): $920 \quad 910-915$.

921

922

Tylianakis JM, Morris RJ 2017. Ecological networks across environmental gradients. Annual Review of Ecology, Evolution, and Systematics 48:25-48.

924

Valdovinos, F. S., P. Moisset de Espanés, J. D. Flores, and R. Ramos-Jiliberto. 2013. Adaptive foraging allows the maintenance of biodiversity of pollination networks. Oikos 122:907-917. van de Leemput, I. A., T. P. Hughes, E. H. van Nes, and M. Scheffer. 2016. Multiple feedbacks and the prevalence of alternate stable states on coral reefs. Coral Reefs 35:857-865.

930

931 Van Wilgenburg, E., G. Driessen, and L. W. Beukeboom. 2006. Single locus complementary sex 932 determination in Hymenoptera: an" unintelligent" design? Frontiers in Zoology 3:1.

934 Vanbergen, A. J. 2013. Threats to an ecosystem service: pressures on pollinators. Frontiers in 935 Ecology and the Environment 11:251-259. 
Williams, N. H., and C. H. Dodson. 1972. Selective attraction of male euglossine bees to orchid floral fragrances and its importance in long distance pollen flow. Evolution:84-95.

Williams, N. M., E. E. Crone, T. a. H. Roulston, R. L. Minckley, L. Packer, and S. G. Potts. 2010. Biological Conservation 143:2280-2291.

950 bees' responses to anthropogenic disturbance. Ecology 90:2068-2076.

Wissel, C., 1984. A universal law of the characteristic return time near thresholds. Oecologia, 65(1), pp.101-107.

956 D.A., Perry, G.L., Richardson, S.J. and Smaill, S.J., 2019. Understanding and Managing SocialEcological Tipping Points in Primary Industries. BioScience 69(5): 335-347.

960 Zayed, A., and L. Packer. 2005. Complementary sex determination substantially increases 961 extinction proneness of haplodiploid populations. Proceedings of the National Academy of 962 Sciences of the United States of America 102:10742-10746. 
972

973

974

975

976

977

978

979

980

981

982

983

984

985

986 